\title{
Effect of Microwave Cooking on Quality of Riceberry Rice (Oryza sativa L.)
}

\author{
Lyda Chin, Nantawan Therdthai $(\mathbb{D}$, and Wannasawat Ratphitagsanti \\ Department of Product Development, Faculty of Agro-Industry, Kasetsart University, Bangkok 10900, Thailand \\ Correspondence should be addressed to Nantawan Therdthai; faginwt@ku.ac.th
}

Received 9 October 2019; Revised 8 August 2020; Accepted 13 August 2020; Published 28 August 2020

Academic Editor: María B. Pérez-Gago

Copyright (c) 2020 Lyda Chin et al. This is an open access article distributed under the Creative Commons Attribution License, which permits unrestricted use, distribution, and reproduction in any medium, provided the original work is properly cited.

\begin{abstract}
Microwaves have been applied for cooking, warming, and thawing food for many years. Microwave heating differs from conventional heating and may cause variation in the food quality. This study determined the quality of Riceberry rice (Oryza sativa L.) after microwave cooking using various rice-to-water ratios at three power levels $(360,600$, and $900 \mathrm{~W})$. The texture of all microwave-cooked samples was in the range $162.35 \pm 5.86$ to $180.11 \pm 7.17 \mathrm{~N}$ and was comparable to the conventionally cooked rice $(162.03 \mathrm{~N})$. The total phenolic content (TPC) and the antioxidant activity of the microwave-cooked rice were higher than those of the conventional-cooked rice. Microwave cooking appeared to keep the TPC in the range 241.15-246.89 mg GAE/100 g $\mathrm{db}$ and the antioxidant activities based on DPPH and ABTS assays in the ranges 134.24-137.15 and 302.80-311.85 mg.TE/100 g db, respectively. Microwave cooking also maintained similar contents of fiber, ash, and total starch to those from conventional cooking. The glycemic index (GI) for all freshly cooked rice samples was not significantly different, and the rice was classified as a high-GI food. Microwave cooking could be recommended as an alternative technique for rice cooking due to its rapid heating regime and the comparable quality and maximized TPC and antioxidant activity of the cooked rice.
\end{abstract}

\section{Introduction}

Rice (Oryza sativa L.) is a vital staple food that is grown in many countries including Thailand, which is known as one of the highest rice producers. Thai rice has a great variety of colors, ranging from black or dark purple to ruby red, golden brown, and white. Regular consumption of some varieties containing high antioxidant activities can benefit human health. Riceberry rice is a new crossbreed between Jao Hom Nin rice and Jasmine rice 105. It is a pigmented rice which has a deep purple whole grain with a unique aroma. Recently, Riceberry rice has become popular for local consumption due to its unique cooked grain characteristics such as fluffy texture, flavor, nutritional value, and health-promoting effects such as anti-inflammatory, anticancer, hyperlipidemic, and hypoglycemic $[1,2]$. Due to its healthenhancing substances, Riceberry rice has gained attention from researchers and food manufacturers as a waste product (Riceberry bran) and as flour and whole grain. It has been used for developing new, natural, and healthy food and commercial food products, as well as in dietary supplements, cosmetics, and pharmaceuticals $[3,4]$.

To cook grains into a palatable, pleasant, and digestible form, rice needs to be boiled or steamed to accomplish suitable starch gelatinization and water absorption. Since people in different countries have different preferences, the cooking methods vary, resulting in two primary cooking techniques. The first involves cooking in large amounts of water with subsequent drainage, commonly referred to the excess or the American method, while the other is based on cooking the rinsed rice in a measured amount of water, commonly known as the Pilaf or the oriental method or the water-absorption method [5]. Europeans mostly prefer to cook rice in excess water, while Asians typically use a rice cooker with a limited amount of water.

Tamura et al. [6] mentioned that eating quality was not only affected by grain characteristics inherent in different cultivars, but was also related to cooking procedures such as the rice-to-water ratio and the cooking temperature. Over time, many heating appliances have evolved including three- 
stone fires, electric cookers, microwave ovens, induction cookers, and other high-tech appliances. Shinde et al. [7] reported $10-25 \%$ variation in the operation efficiency of conventional cooking. Currently, more emphasis is placed on energy conservation, being environmental friendly, and on hygienic production; thus, microwave heating has become more attractive than traditional heating. Microwaves are known to generate heat inside the penetrated medium because of the induced molecular friction in an alternating electromagnetic field. The increased interest in microwaving foods has been encouraged by its faster heating features, lower energy consumption, convenience, and maintenance of nutritional integrity [8]. The $\mathrm{Ca}, \mathrm{K}, \mathrm{Mg}, \mathrm{P}$, and $\mathrm{Zn}$ contents of jalo and black bean species increased after domestic microwave cooking, while the contents of $\mathrm{Cu}, \mathrm{Fe}$, and $S$ did not change [9]. The antioxidant activity of some vegetables such as Momordica charantia and Moringa oleifera could be also enhanced by microwave heating [10]. Microwaves are mostly used for drying, blanching, and pasteurization in food industries. The catering industry uses microwave ovens mainly for reheating preprepared products [11]. Recently, traditional thermal processing has been replaced by microwave cooking due to the accelerated pace associated with the modern lifestyle.

Conventional cooking using an electric cooker involves heating as a combination of conduction (between the heater and the bottom of the container), convection (between the liquid phase and the container), and convection (between the water and rice grains). In contrast, microwave cooking generated heat by dipolar rotations and ionic interactions $[5,12,13]$. These different heating mechanisms could affect the quality of the cooked food. For example, the firmness and chewiness of steamed nonglutinous rice were higher than those from boiling in an electric cooker. Interestingly, the anthocyanins in pigmented rice decreased by around $80 \%$ after cooking in an electric cooker, whereas the phenolic compounds declined by 54\% [4]. Ayimbila and Keawsompong [1] demonstrated that the slow digestion of the starch in Riceberry rice was accelerated depending on the cooking method. The differences in the starch fractions were attributed to the processing conditions and variation in the amount of water, which played a crucial role in starch gelatinization and retrogradation mechanisms during cooking and storage. Besides, the hydrolysate of Riceberry rice significantly enhanced the growth of probiotic strains during in vitro fermentation.

Chatthongpisut et al. [14] reported that the degradation pathway of anthocyanins under microwave heating was different from conventional heating, resulting in different degraded products. Although extremely high-temperature treatment promoted the degradation of anthocyanins in Thai purple rice, it generated phenolic compounds such as protocatechuic acid (PCA), vanillic acid, and ferulic that had an antiproliferative effect on Caco-2 cells. After cooking, the concentration of PCA significantly increased, approximately 1.21-3 times, compared with the raw rice. Yamuangmorn et al. [4] reported that the antioxidant capacity of cooked, purple, nonglutinous rice contrasted in its anthocyanin concentration, indicating that there could be a higher concentration of other antioxidant compounds after cooking. For this reason, cooking Riceberry rice using microwaves could result in different profiles of anthocyanins, phenolic compounds, and biological activity, compared with using an electric cooker.

Therefore, this study aimed to evaluate possible alternative cooking methods for Riceberry rice in a domestic microwave oven and the effect of microwave cooking on antioxidant activities and qualities. The study outcomes would facilitate decisions on cooking rice to maximize its nutrient content for everyday consumption.

\section{Materials and Methods}

2.1. Materials and Chemical Reagents. The Riceberry rice used was a commercial product purchased from a local market in Thailand and stored in a cool room until use. A domestic microwave oven with a frequency of $2,450 \mathrm{MHz}$ (MP-94825S, LG Electronics Inc., Korea) and an electric cooker (Model Sharp KSH-D06, Federal Electric Corp., Ltd, Thailand) were used for this study.

All chemical reagents consisting of gallic acid, Folin-Ciocalteu reagent, 2,2-diphenyl-1-picrylhydrazyl (DPPH), 6-hydroxy-2,5,7,8-tetramethylchromane-2-carboxylic acid (Trolox), 2,2-azino-bis-3 ethylbenzothiazoline6-sulphonic acid diammonium salt (ABTS), methanol, and ethanol were of analytical grade and purchased from SigmaAldrich (St. Louis, MO, USA).

\subsection{Sample Preparation and Rice Cooking. Cooked rice was} prepared in three steps, namely, washing, cooking, and warming. A classical cooking procedure for rice was chosen for the preliminary study. Rice grains were washed three times ( 1 min each time) to eliminate all dirt on the grains. Two different cooking methods were used: microwave cooking using a domestic microwave oven and conventional cooking using an electric cooker. When the cooker automatically shifted to the warm setting, the rice was held for $10 \mathrm{~min}$ at that setting according to the cooker manual, whereas the rice was allowed to stand for $5 \mathrm{~min}$ after $\mathrm{mi}$ crowave cooking.

The experiment used a completely randomized design. There were nine treatments involving either conventional cooking or microwave cooking. For conventional cooking, $100 \mathrm{~g}$ of Riceberry rice grains were prepared in a cooker. Rice-to-water ratios of $1: 1.8,1: 2.0$, and $1: 2.2(\mathrm{w}: \mathrm{v})$ were preliminarily determined to obtain the optimal quality with cooking times of $32 \mathrm{~min}, 34 \mathrm{~min}$, and $37 \mathrm{~min}$, respectively, and designated as EC1, EC2, and EC3, respectively. For microwave cooking, $50 \mathrm{~g}$ of rice was placed in a microwave rice-steaming container (Model Sistema 2.6 L, New Zealand) with a limited water ratio. Then, the rice-steaming container was placed on the center of the turntable plate in the microwave chamber and heated using three power levels $(360 \mathrm{~W}, 600 \mathrm{~W}$, and $900 \mathrm{~W})$. Rice-to-water ratios of $1: 2.8$ (L-MC1) and 1:3.0 (L-MC2) were used for microwave cooking at $360 \mathrm{~W}$. At $600 \mathrm{~W}$, the rice-to-water ratio was 
increased to two levels of $1: 4.8(\mathrm{M}-\mathrm{MC} 1)$ and $1: 5.0$ (M$\mathrm{MC2}$ ). With the maximum power at $900 \mathrm{~W}$, the rice-towater ratio was further increased to $1: 7.0$ (H-MC1) and 1: 7.2 (H-MC2). The variation in the rice-to-water ratio applied was to balance evaporation loss during cooking. The cooking time for each condition was varied to yield palatable cooked rice (Table 1). Rice samples were confirmed to be completely cooked if there was no white core in the rice grains when compressed between two glass plates. The cooked rice was immediately freeze-dried. Freeze-dried samples were ground and kept at $-18^{\circ} \mathrm{C}$ for chemical analysis. The physical quality was analyzed in the freshly cooked form.

\subsection{Determination of Cooking Profiles and Texture of Cooked Rice}

2.3.1. Determination of Temperature Profile and Water Uptake during Cooking. The temperature profile and water uptake during cooking were observed every 2 min until the rice was cooked. The rice and water temperature was measured using a K-type thermocouple device connected to a data logger (Digicon DP-72 Digital Thermometer, Thailand). The water uptake was determined by checking the weight of the rice [15]. The weight of the rice before cooking $\left(\mathrm{W}_{1}\right)$ was recorded. Twenty samples were prepared and cooked individually for different times to obtain palatable eating quality (Table 1). After cooking, each rice sample was left for $30 \mathrm{~s}$ on a filter paper to remove surface water before being reweighed $\left(\mathrm{W}_{2}\right)$. The water uptake ratio was calculated using the following equation:

$$
\text { water uptake ratio }=\frac{\mathrm{W}_{2}}{\mathrm{~W}_{1}} .
$$

Experiments for determination of both the temperature profile and water uptake were carried out based on two replications.

2.3.2. Determination of Texture Profiles of Cooked Rice. The texture of the cooked rice was analyzed using a texture analyzer (Model TA. XT. plus, Stable Micro Systems, UK), following the method by $\mathrm{Wu}$ et al. [16] with some modifications. Twenty cooked rice grains were arrayed on the platform and tested for $5 \mathrm{~min}$ after cooking had finished. A two-cycle compression program (TPA) was run with speeds of $0.5,0.5$, and $5 \mathrm{~mm} / \mathrm{s}$ for the pretest, test, and posttest, respectively. A cylindrical probe $(\mathrm{P} / 75)$ was used to achieve $60 \%$ compression. The experiment was repeated 10 times for each sample. The textural parameters of TPA curves were calculated using the Texture Expert Excede Version 1.0 software (Stable Micro System Software, UK).

\subsection{Comparison of Chemical Qualities from Conventional Cooking and Microwave Cooking}

2.4.1. Determination of Total Phenolic Content and Antioxidant Activities. Free phenolic compounds were extracted using the method by Wiriyawattana et al. [2]. A ground sample was homogenized in $85 \%(\mathrm{v} / \mathrm{v})$ methanol with a ratio of $1: 10 \mathrm{w} / \mathrm{v}$ under shaking at room temperature for $30 \mathrm{~min}$. After centrifugation (Model Rotina 380R, Hettich, Tuttlingen, Germany) at 3,000 rpm for $10 \mathrm{~min}$, the supernatant was decanted. The precipitate was re-extracted. The supernatant samples collected from three extractions were combined and made up using a solvent (methanol $85 \% \mathrm{v} / \mathrm{v}$ ) to the final volume of $50 \mathrm{~mL}$. The extract was used to determine the total phenolic content (TPC) and antioxidant activities using the DPPH radical scavenging assay and the ABTS radical scavenging assay. Extractions were conducted in duplicate.

The amount of TPC was determined using the Folin-Ciocalteu colorimetric method. The extract $(200 \mu \mathrm{L})$ was mixed with $1.5 \mathrm{~mL}$ of sodium carbonate $(75 \mathrm{~g} / \mathrm{L})$ and $1.5 \mathrm{~mL}$ of the Folin-Ciocalteu solution $(1: 10 \mathrm{v} / \mathrm{v})$. The mixed solution was incubated in the dark at a room temperature for $2 \mathrm{~h}$. The absorbance was read using a UV-spectrophotometer (Model 160A, Shimadzu, Japan) at $725 \mathrm{~nm}$. Gallic acid was used as a standard. The results were expressed as milligrams of the gallic acid equivalent per $100 \mathrm{~g}$ of the dry sample (mg $\mathrm{GAE} / 100 \mathrm{~g} \cdot \mathrm{db}$ ).

For the DPPH radical scavenging activity assay, the extract $(500 \mu \mathrm{L})$ was added with $3 \mathrm{~mL}$ of $0.1 \mathrm{mM} \mathrm{DPPH}$ methanol solution. The solution was kept at room temperature for $30 \mathrm{~min}$ before the absorbance was measured at $517 \mathrm{~nm}$ using the UV-spectrophotometer. The DPPH radical scavenging activity was calibrated based on a standard curve of Trolox and expressed as milligrams of the Trolox equivalent per $100 \mathrm{~g}$ of the dry sample $(\mathrm{mg} \mathrm{TE} / 100 \mathrm{~g} \mathrm{db}$ ).

The ABTS radical scavenging activity was determined according to Chatthongpisut et al. [14] with some modifications. A sample of the extract $(100 \mu \mathrm{L})$ was mixed with $3.9 \mathrm{~mL}$ of the working solution. The absorbance was measured at $734 \mathrm{~nm}$ immediately after keeping in the dark for $6 \mathrm{~min}$. The results were expressed as milligram of the Trolox equivalent per 100 grams of the dry sample (mg TE/ $100 \mathrm{~g} \cdot \mathrm{db})$.

2.4.2. Proximate Analysis. The content of crude protein, fat, fiber, moisture, and ash in cooked rice samples was determined according to the Association of Official Analytical Chemists (AOAC) standard methods. The carbohydrate content was calculated by subtracting the moisture, protein, fat, fiber, and ash contents of cooked rice from 100 [17]. All analyses were conducted in triplicate. These results were expressed on a dry weight basis.

2.4.3. Total Starch and Estimated Glycemic Index. The total starch content of each sample was determined using a total starch assay kit (Megazyme International Ireland Ltd., Ireland). The released glucose was measured as the absorbance of the coloration using the UV-spectrophotometer at $510 \mathrm{~nm}$. Total starch (\%) was expressed on a dry weight basis [18].

Starch digestion was determined using a rapid in vitro digestibility assay based on glucometry [19]. The glucose 
TABLE 1: Experimental conditions for rice cooking.

\begin{tabular}{lccc}
\hline Cooking method & Power level $(\mathrm{W})$ & Rice-to-water ratio $(\mathrm{w}: \mathrm{v})$ & Cooking time $(\mathrm{min})$ \\
\hline EC1 & - & $1: 1.8$ & 32 \\
EC2 & - & $1: 2.0$ & 34 \\
EC3 & - & $1: 2.2$ & 37 \\
L-MC1 & 360 & $1: 2.8$ & 30 \\
L-MC2 & 360 & $1: 3.0$ & 31.5 \\
M-MC1 & 600 & $1: 4.8$ & 30 \\
M-MC2 & 600 & $1: 5.0$ & 31 \\
H-MC1 & 900 & $1: 7.0$ & 30 \\
H-MC2 & 900 & $1: 7.2$ & 30.5 \\
\hline
\end{tabular}

EC: conventional cooking using an electric rice cooker, L-MC: microwave cooking using low power level, M-MC: microwave cooking using medium power level, and H-MC: microwave cooking using high power level; 1: low water ratio, 2: medium water ratio, and 3: high water ratio.

concentration in the digesta was measured using a blood glucometer (Model Easymax, Hsinchu Science Park, Taiwan) after a specific time $(0,10,20,30,45,60,90,120,150$, 180,210 , and $240 \mathrm{~min}$ ). Digested starch (DS) per $100 \mathrm{~g}$ dry starch was calculated using the following equation:

$$
\mathrm{DS}=\frac{0.9 \times \mathrm{G}_{\mathrm{G}} \times 180 \times \mathrm{V}}{\mathrm{W} \times \mathrm{S}[100-\mathrm{M}]},
$$

where $G_{G}$ is the glucometer reading $(\mathrm{mM} / \mathrm{L}), V$ is the volume of the digesta $(\mathrm{mL}), 180$ is the molecular weight of glucose, $\mathrm{W}$ is the weight of the sample $(\mathrm{g}), \mathrm{S}$ is the starch content of the sample ( $\mathrm{g} / 100 \mathrm{~g}$ sample), and 0.9 is the stoichiometric constant for starch from glucose contents.

The hydrolysis index of each sample was calculated by dividing the area under its digestogram by the area under the digestogram of white bread. Single-point measurement of starch digestion at $90 \mathrm{~min}$ (HI90) in the samples was also used to calculate the glycemic index (GI). Hence, using the parameter of the modified first-order kinetic model for both the rice sample and white bread (reference), the average GI $\left(\mathrm{GI}_{\mathrm{avg}}\right)$ for each sample was calculated using the following equation [18]:

$$
\mathrm{GI}_{\mathrm{avg}}=\left[\frac{\left(\left(39.21+0.803 \mathrm{HI}_{90}\right)+(39.51+0.573 \mathrm{HI})\right)}{2}\right] \text {, }
$$

where $\mathrm{HI}_{90}$ is hydrolysis at $t=90 \mathrm{~min}$ and $\mathrm{HI}$ is hydrolysis at $t=120 \mathrm{~min}$.

2.5. Statistical Analysis. Data were analyzed using one-way analysis of variance in the SPSS version 12.0 for Windows software (SPSS Inc., Chicago IL, USA). Duncan's test was used to determine significant differences among means $(P \leq 0.05)$.

\section{Results and Discussion}

3.1. Effect of Rice-to-Water Ratio and Cooking Method on Cooked Rice Quality. With the range of rice-to-water ratios used in conventional cooking, the rice grains required $10 \mathrm{~min}$ to reach the maximum temperature of $97.85^{\circ} \mathrm{C}$ compared to only $6 \mathrm{~min}$ using microwave cooking
(Figure 1). The rate constant ( $k$-value) of the temperature increase was significantly different between the cooking methods. However, the rice-to-water ratio did not have a significant effect on the temperature increase during conventional cooking. Likewise, under the same microwave power, increasing the rice-to-water ratio did not affect the rate constant $(P>0.05)$. The $k$-value decreased from $14.67^{\circ} \mathrm{C} \cdot \mathrm{min}^{-1}$ to $13.51^{\circ} \mathrm{C} \cdot \mathrm{min}^{-1}$ when the microwave power decreased from $900 \mathrm{~W}$ to $360 \mathrm{~W}$. Although the rate constant at high and low power varied, the difference was much smaller than those between microwave cooking and conventional cooking probably due to the variation in heating modes. In the current study, the results indicated that high microwave power required a greater water content for cooking than low microwave power due to the increased evaporation at high power. Water is a dipolar molecule that can absorb microwave energy and transform it into heat due to its polar interaction. During the heating process, the vapor pressure is increased, contributing greatly to vapor loss from the system $[13,20]$. Gavahian et al. [12] reported that the higher evaporation rate from microwave cooking led to a greater rate of temperature increase than from the traditional method.

In the water uptake profiles (Figure 2), the cooking method had a significant effect on the rate constant, except for EC3 $\left(k=0.033 \mathrm{~min}^{-1}\right)$ and L-MC1 $\left(k=0.035 \mathrm{~min}^{-1}\right)$. This was possibly due to the slight variations in the rice-to-water ratio (from 2.2 to 2.8 ) and heating power $(300 \mathrm{~W}$ from the electric cooker and $360 \mathrm{~W}$ from the microwave oven). However, the rapid water uptake with microwave cooking at medium and high power could have been related to the initial water content and the cooking temperature because starch begins to gelatinize only when the water content in the rice grain is above the critical water content [21].

With microwave cooking, an increase in the rice-towater ratio did not significantly affect the rate constant of water uptake. The hydration rates increased after increasing the microwave power from low to medium and high levels, indicating that medium and high power resulted in rapid hydration of the rice grains. This was supported by the temperature profile results (Figure 1). The temperature increases for the medium and high power levels were significantly higher than for the low power level. Likewise, He 


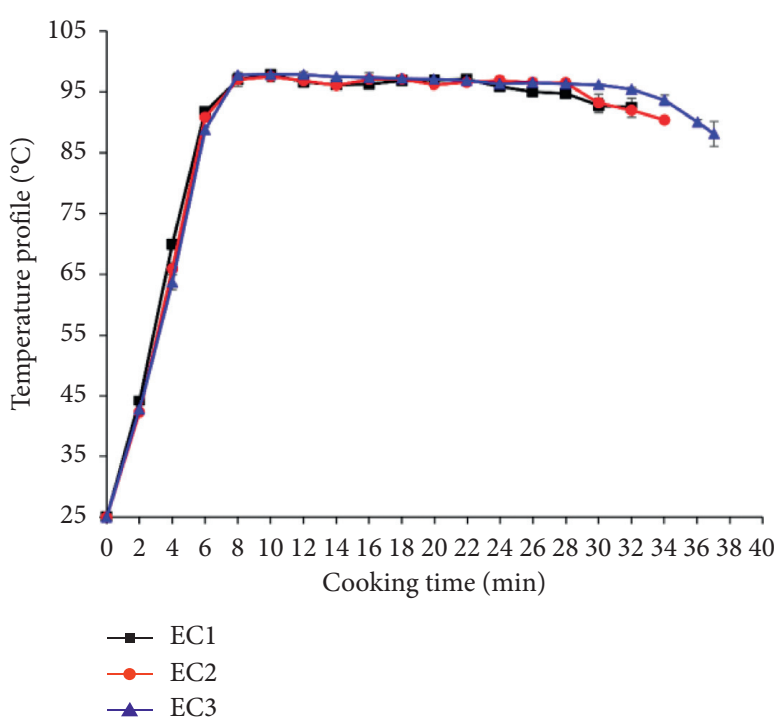

(a)

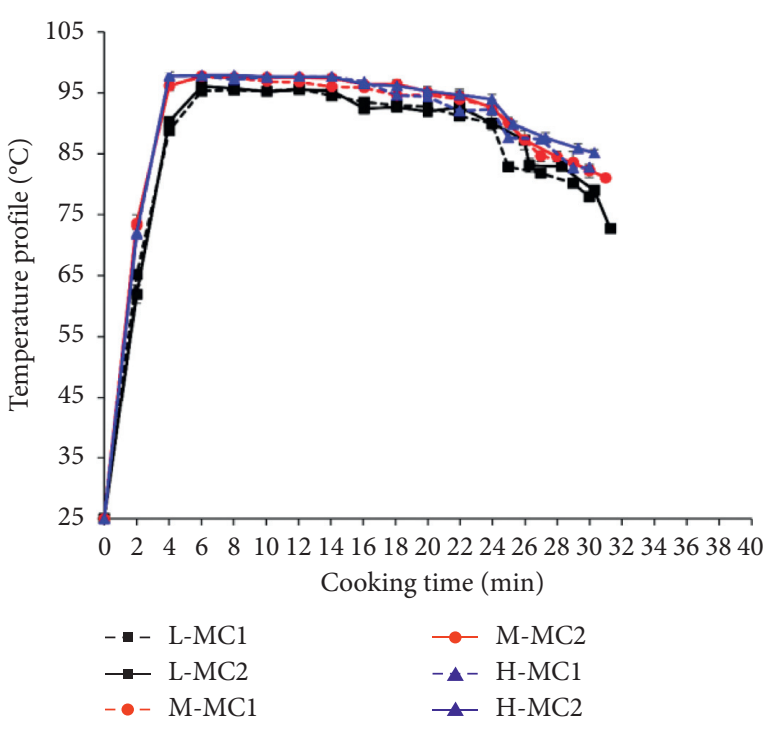

(b)

Figure 1: Temperature profile during conventional cooking (a) and microwave cooking (b). EC: conventional cooking using an electric rice cooker, L-MC: microwave cooking using low power level, M-MC: microwave cooking using medium power level, and H-MC: microwave cooking using high power level. 1: low water ratio, 2: medium water ratio, and 3: high water ratio.

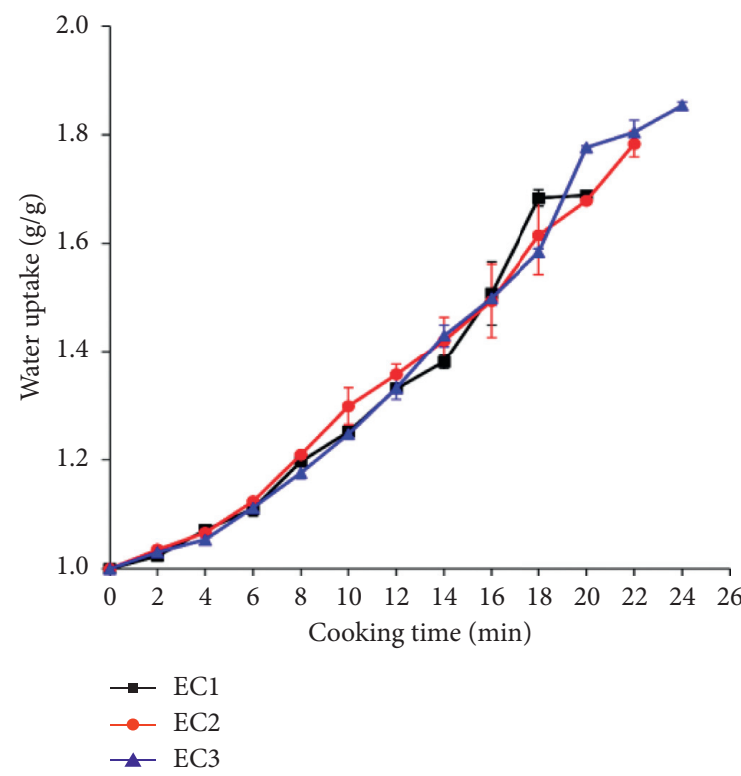

(a)

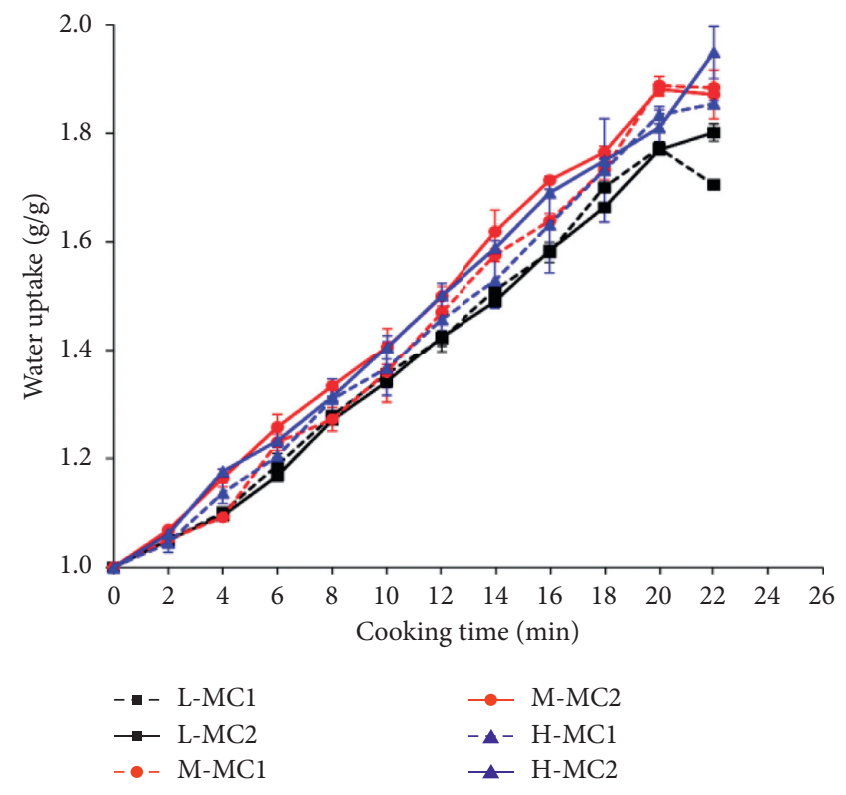

(b)

Figure 2: Water uptake during conventional cooking (a) and microwave cooking (b). EC: conventional cooking using an electric rice cooker, L-MC: microwave cooking using low power level, M-MC: microwave cooking using medium power level, and H-MC: microwave cooking using high power level. 1: low water ratio, 2: medium water ratio, and 3: high water ratio.

et al. [22] mentioned that water absorption in cooked rice was influenced by the cooking temperature. The amount of water uptake by starch granules increased at high temperature due to the effects of the crystalline structure of the granules, the breaking of hydrogen bonds, and the release of amylose and amylopectin into the surrounding area [12]. These results were in agreement with other studies that claimed that the water diffusion rate was related to an increase in temperature, whereas the initial water content and the temperature were the principle factors influencing starch gelatinization [13].

Due to the nonsignificant difference in the rate constant of the temperature profile and water uptake between conventional cooking and the recommended cooking condition 
from the Riceberry rice producer, EC3 was set as the control and the benchmark for the texture of cooked Riceberry rice. Various microwave cooking settings were carried out to find the conditions that could provide a similar texture to EC3. The texture profiles of cooked rice from conventional and microwave cooking are shown in Table 2.

EC3 had values for hardness, adhesiveness, springiness, cohesiveness, and chewiness of $162.03 \mathrm{~N},-671.58 \mathrm{~g} . \mathrm{sec}, 0.40$, 0.46 , and 30.68 , respectively (Table 2 ). Cooked rice obtained from low rice-to-water ratios for each power level (L-MC1, $\mathrm{M}-\mathrm{MC} 1$, and $\mathrm{H}-\mathrm{MC} 1$ ) was not significantly different in hardness. In contrast, the use of high rice-to-water ratios (LMC2, M-MC2, and H-MC2) produced similar texture profiles to that from EC3. Cooked rice with a high ratio of water produced a significantly soft texture. The hardness result was attributed to both the amylose content and the amylose molecular size. Li et al. [23] reported that the hardness of cooked rice was determined by the inner structure of the rice grain, especially the proportion of amylose branches with a degree of polymerization (DP: the average number of monomeric units per molecules) from 1,000 to 2,000 . Thus, a smaller amylose molecular size and a higher proportion of amylose branches accelerated hardness, as these structural features affected the amylose leaching and the degree of starch granule swelling during cooking.

In the current study, the microwave-cooked rice had higher adhesiveness than from conventional cooking, suggesting that microwaving leached more components (total solid loss, starch, amylose, and amylopectin) than from conventional cooking because the microwave energy could weaken the interaction between the amylose and amylopectin and improve their dispersion and separation. Li et al. [23] mentioned that stickiness of cooked rice was affected by the total amount of amylopectin, the proportion of short amylopectin chains, and the amylopectin molecular size in the leachate. Furthermore, the protein content in rice was considered as a secondary factor affecting the starch molecular structure and was related to cooked rice adhesiveness [5].

Conventionally cooked rice had the lowest springiness. Cooking at low and medium microwave power (L-MC1, L-MC2, M-MC1, and M-MC2) produced no significant differences in springiness, though at high microwave power (H-MC1), springiness was higher than that for H-MC2 (Table 2). Therefore, the rice-to-water ratio may influence springiness only under high-power conditions. Cohesiveness is the internal force holding rice together and indicates the amount of compression before breaking. Microwave cooking at high power (H-MC2) tended to result in similar cohesiveness (0.47) and chewiness (36.15) to those obtained from the control (Table 2). It was noticeable that cohesiveness and chewiness values decreased with an increase in the rice-to-water ratio, and these values varied depending on the rice-to-water ratios for both low and high power of microwave cooking. These results were in agreement with the findings by Chusak et al. [24] that cohesiveness depended on the nature of the food and the external factors such as the moisture content and the temperature. The microwave-cooked rice had a low chewiness value, similar to the conventionally cooked rice, when the high initial rice-to- water ratio was used with high microwave power. By decreasing the rice-to-water ratio or the microwave power, the chewiness was increased.

Alteration of the textural characteristics of cooked rice by microwave cooking might be attributed to the reduction in the degree of starch gelatinization [24]. The different heating patterns could cause some modifications in the physical structure of cooked rice. Moreover, the rate of temperature increase may affect the kinetic constant, the rate of starch hydrolysis, and the texture and pasting properties due to differences in the starch microstructure $[12,25]$. The texture of cooked rice was influenced by the availability of water during the early stage of cooking, which determined the hydration of the protein and the concentration of the dispersed and viscous phases of the starch [26].

Based on no significant difference in hardness and the similarity of the other texture attributes, the options using microwave cooking with high initial rice-to-water ratios (LMC2, M-MC2, and H-MC2) were selected for further study.

\subsection{Comparison of Microwave Cooking and Conventional Cooking}

3.2.1. Changes in Total Phenolic Content and Antioxidant Activity after Cooking. After cooking under both conventional and microwave conditions, the TPC of cooked rice (214.41-246.89 mg GAE/100 g db) decreased substantially compared to that of the rice grain $(302.22 \mathrm{mg}$ GAE/ $100 \mathrm{~g}$ $\mathrm{db}$ ), as shown in Table 3. Conventional cooking (EC3) resulted in a significant loss of $29.05 \%$ in the TPC in the cooked rice, but the TPC loss was reduced by 18.31, 20.21, and $18.86 \%$ for L-MC2, M-MC2, and H-MC2, respectively.

The free radical scavenging activity of cooked rice was measured using two different methods (DPPH and ABTS assays), to reduce the interference by other substances in the test. The scavenging activities of rice grains were 157.09 and $407.03 \mathrm{mg}$.TE/100 $\mathrm{g} \mathrm{db}$ for DPPH and ABTS, respectively. The DPPH scavenging activity of the cooked rice (117.01-137.15 mg.TE/100 g db) was lower than ABTS scavenging activity (230.05-311.85 mg.TE/100 g db), as shown in Table 3 . The lower activity in the DPPH assays may have been due to chemical mechanisms. The DPPH assay generally measures hydrophilic antioxidants, while the ABTS assay determines both lipophilic and hydrophilic antioxidants [27]. Compared to rice grains, the loss of DPPH and ABTS activities in conventionally cooked rice was 25.52 and $43.48 \%$, whereas for microwave cooking, the ranges were $12.69-14.55 \%$ and $23.38-25.61 \%$, respectively. There was no significant difference in the TPC and antioxidant activities of the cooked rice when cooked at either low or high microwave power. The decrease in the antioxidant activities after cooking was consistent with the reduction in the TPC. These results indicated that the TPC contributed to antioxidant activities in Riceberry rice. After cooking, the decrease in the TPC and the scavenging activity of conventionally cooked rice was greater than that from microwaving.

The percentage loss could be related to cooking conditions such as the rice-to-water ratio, heating source, and 
TABLe 2: Texture profiles of cooked rice from conventional cooking and microwave cooking.

\begin{tabular}{|c|c|c|c|c|c|}
\hline Treatment & Hardness $(\mathrm{N})$ & Adhesiveness (g.sec) & Springiness $(-)$ & Cohesiveness (-) & Chewiness (-) \\
\hline EC3 & $162.03 \pm 4.08^{\mathrm{b}}$ & $-671.58 \pm 42.64^{\mathrm{a}}$ & $0.40 \pm 0.05^{\mathrm{d}}$ & $0.46 \pm 0.00^{c}$ & $30.68 \pm 3.58^{\mathrm{d}}$ \\
\hline L-MC1 & $180.11 \pm 7.17^{\mathrm{a}}$ & $-741.54 \pm 47.13^{\mathrm{a}}$ & $0.56 \pm 0.07^{\mathrm{ab}}$ & $0.51 \pm 0.03^{\mathrm{a}}$ & $53.62 \pm 11.01^{\mathrm{ab}}$ \\
\hline L-MC2 & $166.72 \pm 4.62^{\mathrm{b}}$ & $-886.57 \pm 42.81^{b}$ & $0.54 \pm 0.05^{\mathrm{b}}$ & $0.49 \pm 0.01^{\mathrm{b}}$ & $44.60 \pm 6.39^{c}$ \\
\hline M-MC1 & $176.13 \pm 5.42^{\mathrm{a}}$ & $-828.66 \pm 81.00^{\mathrm{b}}$ & $0.53 \pm 0.09^{b c}$ & $0.50 \pm 0.01^{\mathrm{ab}}$ & $47.66 \pm 9.82^{b c}$ \\
\hline M-MC2 & $166.35 \pm 7.98^{\mathrm{b}}$ & $-1013.26 \pm 182.22^{\mathrm{c}}$ & $0.53 \pm 0.06^{\mathrm{bc}}$ & $0.49 \pm 0.01^{\mathrm{b}}$ & $44.39 \pm 7.47^{\mathrm{c}}$ \\
\hline $\mathrm{H}-\mathrm{MC} 1$ & $175.21 \pm 8.22^{\mathrm{a}}$ & $-1064.35 \pm 67.01^{\mathrm{cd}}$ & $0.62 \pm 0.08^{\mathrm{a}}$ & $0.50 \pm 0.02^{\mathrm{ab}}$ & $56.20 \pm 12.07^{\mathrm{a}}$ \\
\hline $\mathrm{H}-\mathrm{MC} 2$ & $162.35 \pm 5.86^{\mathrm{b}}$ & $-1118.02 \pm 53.60^{\mathrm{d}}$ & $0.47 \pm 0.04^{\mathrm{c}}$ & $0.47 \pm 0.01^{\mathrm{c}}$ & $36.15 \pm 5.20^{\mathrm{d}}$ \\
\hline
\end{tabular}

Data expressed as mean \pm standard deviation in triplicate; ${ }^{\mathrm{a}-\mathrm{d}}$ different superscript letters indicate a significant $(P \leq 0.05)$ difference. EC: conventional cooking using an electric rice cooker, L-MC: microwave cooking using low power level, M-MC: microwave cooking using medium power level, and H-MC: microwave cooking using high power level. 1: low water ratio, 2: medium water ratio, and 3: high water ratio.

TABLE 3: Total phenolic content and antioxidant activity of the rice grain and the cooked rice from conventional cooking and microwave cooking.

\begin{tabular}{|c|c|c|c|}
\hline Sample & $\begin{array}{l}\text { Total phenolic content } \\
(\mathrm{mg} \text { GAE/100 } \mathrm{g} \mathrm{db})\end{array}$ & $\begin{array}{l}\text { Scavenging activity using DPPH } \\
(\mathrm{mg} \mathrm{TE} / 100 \mathrm{~g} \mathrm{db})\end{array}$ & $\begin{array}{l}\text { Scavenging activity using ABTS } \\
(\mathrm{mg} \mathrm{TE} / 100 \mathrm{~g} \mathrm{db})\end{array}$ \\
\hline Riceberry rice grain & $302.22 \pm 2.47^{\mathrm{a}}$ & $157.09 \pm 0.02^{\mathrm{a}}$ & $407.03 \pm 2.26^{\mathrm{a}}$ \\
\hline EC3 & $214.41 \pm 4.78^{\mathrm{c}}$ & $117.01 \pm 2.64^{c}$ & $230.05 \pm 11.58^{\mathrm{c}}$ \\
\hline L-MC2 & $246.89 \pm 4.68^{b}$ & $134.98 \pm 1.21^{\mathrm{b}}$ & $311.85 \pm 2.90^{\mathrm{b}}$ \\
\hline M-MC2 & $241.15 \pm 2.68^{b}$ & $134.24 \pm 1.13^{b}$ & $304.91 \pm 1.52^{\mathrm{b}}$ \\
\hline H-MC2 & $245.22 \pm 1.97^{\mathrm{b}}$ & $137.15 \pm 0.99^{\mathrm{b}}$ & $302.80 \pm 12.52^{\mathrm{b}}$ \\
\hline
\end{tabular}

Data expressed as mean \pm standard deviation in duplicate; ${ }^{\text {a-c }}$ different superscript letters indicate a significant $(P \leq 0.05)$ difference. EC: conventional cooking using an electric rice cooker, L-MC: microwave cooking using low power level, M-MC: microwave cooking using medium power level, and H-MC: microwave cooking using high power level; 1: low water ratio, 2: medium water ratio, and 3: high water ratio.

cooking time. The decline has also been attributed to the oxidation of malvidin-3-glucoside and malvidin-3,5-diglucosides as a long conventional cooking time $(37 \mathrm{~min})$, and characteristics of individual phenolic compounds could affect the loss of antioxidants [14]. The yield of anthocyanins in the grape skin could be improved by using high microwave energy since heat treatment partially breakdowns an ester bond between the bound phenolic compounds and cell walls to release some free phenolic compounds. According to the degradation pathway of malvidin-3-glucoside and malvidin3,5-diglucoside under microwave treatment, the formation of anthocyanone A, hydroxycoumarins, dihydroxy phenylacetaldehyde, and eight other compounds was detected in the microwave-treated samples at $700 \mathrm{~W}$. In contrast, those compounds were not found in samples heated using a water bath at $98 \pm 2{ }^{\circ} \mathrm{C}$. The direct microwave effect formed hydrogen peroxide, resulting in Baeyer-Villiger-type oxidation, which was the main degradation pathway. With this reaction, anthocyanins were oxidized to form a derivative of the benzoyloxyphenyl acetic acid ester, which was named malvone. Then, malvone was further hydrolyzed to form syringic acid and anthocyanone. At the same time of oxidation, the conventional degradation pathway of anthocyanins also occurred [28]. Therefore, the higher remaining TPC and antioxidant activity in the microwave-cooked rice was attributed to the difference in the degradation pathway and the formation of new degraded products that may not be found in conventionally cooked rice.

Another factor that may attribute to the great loss in conventionally cooked rice was from rice partially stuck and burnt on the bottom of the container used in electric cooking as this would be the hottest area. In contrast, no rice layer was formed or deposited on the microwave rice-steaming container because no hot surface is required for microwave heating. Therefore, component leaching, thermal decomposition, and interaction mainly with the protein content were considered as possible factors responsible for the decrease in the phenolic content of the pigmented rice during conventional cooking. In addition, the presence of sugars and phenolic acids could help to maintain anthocyanin stability [4]. Thus, the effect of cooking process on the degradation mechanism of anthocyanin and phenolic acid should be carefully identified and quantified with the existent compounds in further study.

3.2.2. Changes in Chemical Composition. Changes in cooked rice composition such as the ash, fat, fiber, protein, and carbohydrate contents after different cooking conditions are shown in Table 4. Under all cooking conditions, there were no significant difference in the ash or total dietary fiber contents. However, conventional cooking (control) resulted in the lowest protein content $(11.11 \%)$ of any of the cooking conditions. Notably, there were no significant differences in the protein content among all microwave-cooked rice samples. This indicated that conventional cooking promoted the leaching of protein from rice grains to the cooking water, while microwave cooking slowed down biochemical reactions and some changes in the molecular conformation of the protein [29]. 
TABLE 4: Chemical composition of the Riceberry rice grain (Oryza sativa L.) and the cooked rice from conventional cooking and microwave cooking.

\begin{tabular}{|c|c|c|c|c|c|c|c|}
\hline Sample & Ash content $(\% \mathrm{db})$ & Fat $(\% \mathrm{db})$ & Fiber (\% db) & $\begin{array}{l}\text { Protein } \\
(\% \mathrm{db})\end{array}$ & $\begin{array}{c}\text { Carbohydrate } \\
(\% \mathrm{db})\end{array}$ & $\begin{array}{l}\text { Total starch } \\
(\% \text { in } \mathrm{db})\end{array}$ & Estimated GI \\
\hline Raw & $a$ & 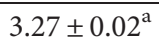 & 2.46 & $12.31 \pm 0.03^{\mathrm{a}}$ & $72.89 \pm 0.03^{\mathrm{d}}$ & - & - \\
\hline EC3 & $14 \pm 0.01^{\mathrm{a}}$ & $3.30 \pm 0.03^{\mathrm{a}}$ & $2.29 \pm 0.07^{\mathrm{a}}$ & $11.11 \pm 0.07^{\mathrm{b}}$ & $78.23 \pm 0.18^{\mathrm{a}}$ & $75.27 \pm 1.84^{\mathrm{a}}$ & $73.00 \pm 0.04^{\mathrm{a}}$ \\
\hline L-MC2 & $1.47 \pm 0.02^{\mathrm{a}}$ & $3.23 \pm 0.03^{\mathrm{a}}$ & $2.44 \pm 0.16^{\mathrm{a}}$ & $12.03 \pm 0.30^{\mathrm{a}}$ & $76.91 \pm 0.16^{c}$ & $74.27 \pm 1.26^{\mathrm{a}}$ & $73.11 \pm 0.43^{\mathrm{a}}$ \\
\hline M-MC2 & $1.45 \pm 0.00^{\mathrm{a}}$ & $3.22 \pm 0.04^{\mathrm{a}}$ & $2.32 \pm 0.09^{\mathrm{a}}$ & $12.11 \pm 0.26^{\mathrm{a}}$ & $77.56 \pm 0.24^{\mathrm{b}}$ & $75.29 \pm 0.84^{\mathrm{a}}$ & $73.01 \pm 0.29^{\mathrm{a}}$ \\
\hline H-MC2 & $1.47 \pm 0.01^{\mathrm{a}}$ & $3.06 \pm 0.10^{\mathrm{b}}$ & $2.32 \pm 0.09^{\mathrm{a}}$ & $12.01 \pm 0.04^{\mathrm{a}}$ & $77.48 \pm 0.13^{\mathrm{b}}$ & $75.90 \pm 0.75^{\mathrm{a}}$ & $73.01 \pm 0.83^{\mathrm{a}}$ \\
\hline
\end{tabular}

Data expressed as mean \pm standard deviation in triplicate; ${ }^{\mathrm{a}-\mathrm{d}}$ different superscript letters indicate a significant $(P \leq 0.05)$ difference. EC: conventional cooking using an electric rice cooker, L-MC: microwave cooking using low power level, M-MC: microwave cooking using medium power level, and H-MC: microwave cooking using high power level. 1: low water ratio, 2: medium water ratio, and 3: high water ratio.

Using high microwave power caused a significant reduction in the fat content of the cooked rice compared with the other conditions. This might have been directly correlated to the loss of the detached oil bodies through a small opening between the paleal and lemma during the hydration of rice [30]. The conventionally cooked rice had a high proportion of carbohydrate $(78.23 \%)$ since its protein content was more affected than by microwave cooking.

The GI is a physiological measure used to classify food rich in carbohydrate, based on the food's potential to raise the blood glucose level. Rice is considered to be a high-GI food (GI > 70). The differences in the starch digestibility of cooked rice have been attributed to the disruption of starch gelatinization, structure modification, breakdown of the cell wall, and protein denaturation [31]. In the current study, the GI values of all cooked rice samples were in the range 73.00-73.11 and were not significantly different. Thus, as the GI was greater than 70, the cooked Riceberry rice was classified as having a high glycemic index. Cooking possibly increased the rate of hydrolysis, and the gelatinized starch of the freshly cooked rice was readily available for the enzymatic attack because cooking increased the space around crystallites through the swelling of starch grains and decreased the continuity in the construction of protein by changing the structure of starch molecules [22, 32]. Thus, cooking using the electric cooker or the microwave oven did not produce a significant change in the GI. This coincided with that reported by Chapagai et al. [33] that there were no GI differences among different cooked rice samples although there were some differences in the amount of amylose-lipid complexes.

\section{Conclusion}

In general, rice is conventionally cooked in an electric cooker. However, by changing to microwave cooking, the rate constant of the temperature profile and increased water uptake during cooking were significantly improved. The initial rice-to-water ratio significantly influenced the texture of the cooked rice. Both microwave cooking and conventional cooking produced similar results for the texture, total starch content, and GI. In terms of bioactive compounds, microwave cooking could retain higher levels of TPC and antioxidant activities in the cooked rice than from conventional cooking. Therefore, microwave cooking could be recommended for Riceberry rice which has an inherent high antioxidant activity. This alternative to the conventional rice cooker method could reduce the cooking time by $14-18 \%$ and preserve nutritional quality. Consumption of Riceberry rice cooked in a microwave oven could maximize health benefits like antioxidant activity while producing a comparable textural quality.

\section{Data Availability}

The data used to support the findings in this study are available from the corresponding author upon request.

\section{Conflicts of Interest}

The authors have no conflicts of interest.

\section{Acknowledgments}

Financial support was provided by the Kasetsart University Research and Development Institute (KURDI), Bangkok, Thailand, and the Department of Product Development and Faculty of Agro-Industry, Kasetsart University.

\section{References}

[1] F. Ayimbila and S. Keawsompong, "Effect of processing procedures on in vitro digestibility and colonic fermentation of Riceberry rice," Journal of Microbiology, Biotechnology and Food Sciences, vol. 8, no. 3, pp. 940-946, 2018.

[2] P. Wiriyawattana, S. Suwonsichon, and T. Suwonsichon, "Effects of drum drying on physical and antioxidant properties of riceberry flour," Agriculture and Natural Resources, vol. 52, no. 5, pp. 445-450, 2018.

[3] M. Peanparkdee, R. Yamauchi, and S. Iwamoto, "Characterization of antioxidants extracted from Thai riceberry bran using ultrasonic-assisted and conventional solvent extraction methods," Food and Bioprocess Technology, vol. 11, no. 4, pp. 713-722, 2017.

[4] S. Yamuangmorn, B. Dell, and C. Prom-u-thai, "Effects of cooking on anthocyanin concentration and bioactive antioxidant capacity in glutinous and non-glutinous purple rice," Rice Science, vol. 25, no. 5, pp. 270-278, 2018.

[5] H. Li and R. G. Gilbert, "Starch molecular structure: the basis for an improved understanding of cooked rice texture," Carbohydrate Polymers, vol. 195, pp. 9-17, 2018.

[6] M. Tamura, T. Nagai, Y. Hidaka, T. Noda, M. Yokoe, and Y. Ogawa, "Changes in histological tissue structure and 
textural characteristics of rice grain during cooking process," Food Structure, vol. 1, no. 2, pp. 164-170, 2014.

[7] Y. H. Shinde, A. Vijayadwhaja, A. B. Pandit, and J. B. Joshi, "Kinetics of cooking of rice: a review," Journal of Food Engineering, vol. 123, pp. 113-129, 2014.

[8] G. Mishra, D. C. Joshi, D. Mohapatra, and V. B. Babu, "Varietal influence on the microwave popping characteristics of sorghum," Journal of Cereal Science, vol. 65, pp. 19-24, 2015.

[9] A. S. T. Ferreira, J. Naozuka, G. A. R. Kelmer, and P. V. Oliveira, "Effects of the domestic cooking on elemental chemical composition of beans species (phaseolus vulgaris 1.)," Journal of Food Processing, vol. 2014, p. 6, Article ID 972508, 2014.

[10] S. Subramaniam, M. H. B. Rosdi, and U. R. Kuppusamy, "Customized cooking methods enhance antioxidant, antiglycemic, and insulin-like properties of Momordica charantia and Moringa oleifera," Journal of Food Quality, vol. 2017, Article ID 9561325, 9 pages, 2017.

[11] A. M. Kalla and R. Devaraju, "Microwave energy and its application in food industry: a reveiw," Asian Journal of Dairy and Food Research, vol. 36, no. 1, pp. 37-44, 2017.

[12] M. Gavahian, Y.-H. Chu, and A. Farahnaky, "Effects of ohmic and microwave cooking on textural softening and physical properties of rice," Journal of Food Engineering, vol. 243, pp. 114-124, 2019.

[13] K. Kanjanapongkul, "Rice cooking using ohmic heating: determination of electrical conductivity, water diffusion and cooking energy," Journal of Food Engineering, vol. 192, pp. 1-10, 2017.

[14] R. Chatthongpisut, S. J. Schwartz, and J. Yongsawatdigul, "Antioxidant activities and antiproliferative activity of Thai purple rice cooked by various methods on human colon cancer cells," Food Chemistry, vol. 188, pp. 99-105, 2015.

[15] B. O. Juliano, The Rice Grain and its Gross Composition, Chemistry and Technologypp. 17-58, Elsevier, Amsterdam, Netherlands, 2nd edition, 1985.

[16] J. Wu, J. Chen, W. Liu et al., "Effects of aleurone layer on rice cooking: a histological investigation," Food Chemistry, vol. 191, pp. 28-35, 2016.

[17] AOAC, Official Methods of Analysis of the Association of Official Analytical Chemists, AOAC, Washington DC, USA, Seventeen edition, 2000.

[18] C. Lerdluksamee, K. Srikaeo, J. A. M. Tutusaus, and J. G. Diéguez, "Physicochemical properties and starch digestibility of Scirpus grossus flour and starch," Carbohydrate Polymers, vol. 97, no. 2, pp. 482-488, 2013.

[19] P. A. Sopade and M. J. Gidley, "A rapid in-vitro digestibility assay based on glucometry for investigating kinetics of starch digestion," Starch-Stärke, vol. 61, no. 5, pp. 245-255, 2009.

[20] H. Ekka, D. Padhee, L. Sharma, M. Diwakar, R. Lahari, and S. Tiwari, "Study of cooking quality of selected varieties of rice at varying temperature using induction heater," International Journal of Food Science and Nutrition, vol. 1, no. 2, pp. 4-7, 2016.

[21] A. Briffaz, P. Bohuon, J.-M. Méot, M. Dornier, and C. Mestres, "Modelling of water transport and swelling associated with starch gelatinization during rice cooking," Journal of Food Engineering, vol. 121, pp. 143-151, 2014.

[22] M. He, C. Qiu, Z. Liao, Z. Sui, and H. Corke, "Impact of cooking conditions on the properties of rice: combined temperature and cooking time," International Journal of Biological Macromolecules, vol. 117, pp. 87-94, 2018.
[23] H. Li, J. Yang, M. Gao, J. Wang, and B. Sun, "Washing rice before cooking has no large effect on the texture of cooked rice," Food Chemistry, vol. 271, pp. 388-392, 2019 a.

[24] C. Chusak, J. A. Y. Ying, J. L. Zhien et al., "Impact of Clitoria ternatea (butterfly pea) flower on in vitro starch digestibility, texture and sensory attributes of cooked rice using domestic cooking methods," Food Chemistry, vol. 295, pp. 646-652, 2019.

[25] L. Yu, M. S. Turner, M. Fitzgerald, J. R. Stokes, and T. Witt, "Review of the effects of different processing technologies on cooked and convenience rice quality," Trends in Food Science \& Technology, vol. 59, pp. 124-138, 2017.

[26] H. Li, J. Yang, S. Yan, N. Lei, J. Wang, and B. Sun, "Molecular causes for the increased stickiness of cooked non-glutinous rice by enzymatic hydrolysis of the grain surface protein," Carbohydrate Polymers, vol. 216, pp. 197-203, 2019 b.

[27] W. Daiponmak, C. Senakun, and S. Siriamornpun, "Antiglycation capacity and antioxidant activities of different pigmented Thai rice," International Journal of Food Science \& Technology, vol. 49, no. 8, pp. 1805-1810, 2014.

[28] M. Zhao, Y. Luo, Y. Li et al., "The identification of degradation products and degradation pathway of malvidin-3-glucoside and malvidin-3, 5-diglucoside under microwave treatment," Food Chemistry, vol. 141, no. 3, pp. 3260-3267, 2013.

[29] S. Zhao, S. Xiong, C. Qiu, and Y. Xu, "Effect of microwaves on rice quality," Journal of Stored Products Research, vol. 43, no. 4, pp. 496-502, 2007.

[30] S. J. Kale, S. K. Jha, G. K. Jha, J. P. Sinha, and S. B. Lal, "Soaking induced changes in chemical composition, glycemic index and starch characteristics of basmati rice," Rice Science, vol. 22, no. 5, pp. 227-236, 2015.

[31] S. Wang, P. Li, T. Zhang, J. Yu, S. Wang, and L. Copeland, "In vitro starch digestibility of rice flour is not affected by method of cooking," Lwt, vol. 84, pp. 536-543, 2017.

[32] M. Tamura, J. Singh, L. Kaur, and Y. Ogawa, "Impact of the degree of cooking on starch digestibility of rice - an in vitro study," Food Chemistry, vol. 191, pp. 98-104, 2016.

[33] M. K. Chapagai, N. A. Bakar, R. A. Jalil et al., "Glycaemic index values and physicochemical properties of five brown rice varieties cooked by different domestic cooking methods," Functional Foods in Health and Disease, vol. 6, no. 8, p. 506, 2016. 